\title{
Inclusivity bugs and the language we use
}

\author{
Mark C. Miller ${ }^{1}$ \\ ${ }^{1}$ Affiliation not available
}

November 3, 2021

If we knew many terms and phrases we commonly use such as, for example, sanity check, grandfathered, ladies and gentlemen or low man on the totem pole can discourage coworkers, colleagues, collaborators and other potential contributors from participating in our projects with their full authentic selves, would we still use them?

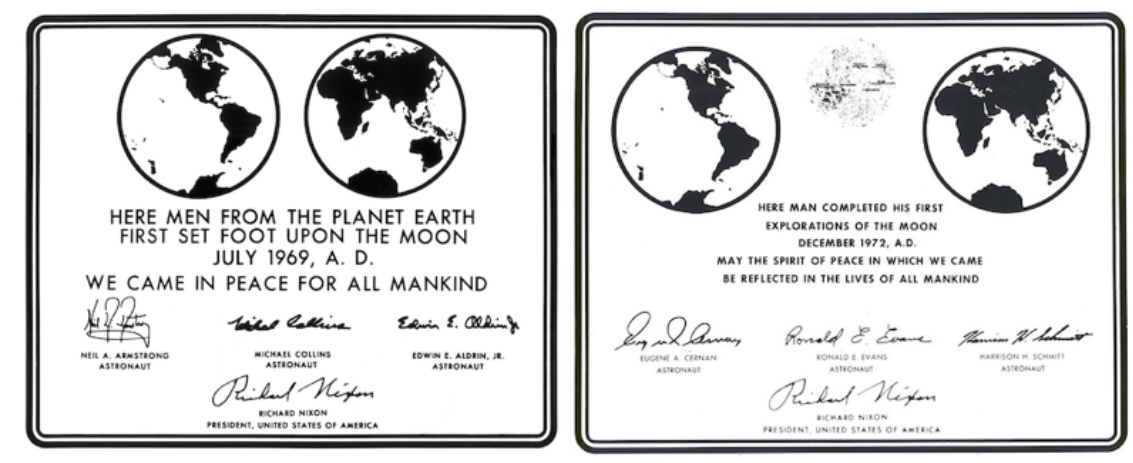

Figure 1: The language used in the plaques left at first and last Moon landing sites.

When Armstrong first set foot upon the Moon, he said "This is a small step for a man but a giant leap for mankind." Minutes later, responding to President Nixon, he said ${ }^{1}$ "It is a great honor... to be here representing... men of peaceable nations, men with an interest and a curiosity, and men with a vision for the future."

Taken out context of the 1960s, the repeated use of man and men certainly doesn't read very much like space exploration included women. More importantly, however, it also didn't sound that way to many of the $\sim 650$ million listeners ${ }^{2}$ at the time. Worse, even current writings about the Apollo space program tend to parrot the same language using phrases like when man first walked on the Moon or what a great achievement it was for mankind.

To help skeptics empathize, imagine if instead of a crew of all white, Christian men as the first Apollo mission was (maybe all Apollo missions), the first mission to Mars is crewed by all Black, Muslim women and the first to walk on Mars says something like "This is a small step for a woman but a giant leap for woman-kind... Allahu Akbar."

Joanna Lee, ${ }^{3}$ a contributor to the Inclusive Naming Initiative ${ }^{4}$ and author of inclusive language guidelines on inclusiveprism.org ${ }^{5}$, calls the use of such language in software projects an inclusivity bug because it creates 
barriers to full participation by all stakeholders. Inclusivity bugs arise out of the use of language that discourages whole groups of people, often from underrepresented and/or historically oppressed populations, from contributing as their full authentic selves. This can happen regardless of how common-place any manner of speaking or turn of phrase is or may have been for its time. In fact, an alarming variety of inclusivity bugs stem from language we all commonly use.

Readers might dismiss Armstrong's specific words as merely a figure of speech or a sign of the times. That's just the way we spoke then. Armstrong didn't intend to exclude women. Or, readers might think that the problem is the English language because it does not easily avail itself to gender neutrality. But, that would be wrong. While English is notoriously lacking in gender-neutral pronouns, it is otherwise one of the most accommodating languages for gender neutrality.

As HPC/CSE software developers maybe we're not doing anything nearly as momentous as being the first Earthlings to walk on another planet. Do we really need to worry so much about inclusive language? If we care about our project's reach and maximizing our ability to attract and retain collaborators, developers, users or sponsors, then yes we do. That is because the language we use has the power to welcome others in as well as push them away.

Software projects involve a lot of communication. As developers, we write code, comments, documentation, research papers, presentations, job postings, promotional materials, discussion posts, emails, chats, tweets and more. It is now well known that language used in job postings ${ }^{6}$ has the effect of biasing the respondent pool. The more we adopt inclusive language, the more we reduce biases and create welcoming and safe spaces for all participants to be their authentic selves, to be productive and to thrive.

Any readers who have written proposals likely already appreciate the role language plays when communicating our work. When writing proposals, we often struggle with and debate at length how individual word choices will align our proposal with the sponsor's call. In other words, we all already appreciate the importance of and can be quite mindful of the impact of language on funding. Being mindful of the impact of language on inclusion is no different.

Inclusive naming is closely related to inclusive language but focuses primarily on the naming of things. That is the names we choose for abstract objects of our software systems. There is probably no other human activity that involves the naming of new things as much as software development. There is power in naming and with great power, comes great responsibility. The names we use in our code and its associated artifacts (PR reviews, documentation, presentations, social media posts, etc.) are often shaped by our culture.

A special challenge with inclusive naming is that people tend to get pretty attached to names. Was Edmund Halley the first to observe and record the comet ${ }^{7}$ that bears his name ${ }^{8}$ Chinese astronomers observed and recorded $^{9}$ it almost 2,000 years earlier! Was Pythagoras the first person ${ }^{10}$ ever to discover and write down the rule we know as the Pythagorean theorem? Plenty of scholarly research ${ }^{11}$ says others developed it earlier.

For many terms and phrases in common use, we are simply unwittingly parroting others. That can be problematic when one is not mindful of the meaning, history or impact of a term. For example, are readers fully aware of why we call defects in software bugs? ${ }^{12}$ How might women feel reading documentation that constantly refers to the user as he, his or him ? $^{13}$ How might Black people feel reading that existing users will be grandfathered $i n^{14}$ when the software license terms are changed? How might neurodiverse people feel about being tasked to add sanity checks ${ }^{15}$ to a software package's test suite?

By the way, do readers recognize any bias in the preceding questions? It's subtle but worth mentioning. The way these questions are worded assume that only women would care about documentation that is laden with male pronouns or that only Black people would care about language derived out of racially oppressive practices or that only neurodiverse people would care about language associated with atypical patterns of thought or behavior. The truth is, we all (should) care. And, to use language that assumes or suggests otherwise is, well, not inclusive.

No way to phrase something will be acceptable to literally every individual. And, there is no reason we 
should be aiming for that either. Catering to any one individual's tastes and sensibilities is not what inclusive language is all about. The goal of inclusive language is to reduce the use of terms and phrases that discourage whole groups of people from participating as their full authentic selves. It is not about being politically correct or being the language police. It is not about avoiding offending ${ }^{16}$ people either. It's about being willing to acknowledge that certain terms and phrases (and, honestly, even names, icons and logos), however common in current culture, can be unnecessarily exclusive and being willing to consider and adopt alternatives that are less so.

If you've read this far, you may be asking yourself, how do I get started? We suggest reading some of the resources available from other major organizations such as the federal government's Plain Language ${ }^{17}$, Google's Inclusive Style ${ }^{18}$ or Microsoft's Bias Free Communication. ${ }^{19}$ Be aware that some resources and efforts are focused more upon identifying specific words and phrases to be fixed whereas others are focused more on principles of inclusive language. In particular, if you are looking for tooling to help alert you to inclusive language issues, some resources provide tooling as a web service. That said, community standards and DevOps-hardened tooling for inclusive language similar to spelling or grammar checkers is still in its infancy stages.

Ever since adopting the practice myself, not a week goes by that I don't have the experience of questioning a term or phrase I am about to use. I often spend a few minutes searching the web to learn more about it. This includes terms and phrases I have used many times before without really thinking about it such as describing an off-topic enhancement request as having gone off the reservation ${ }^{20}$ or replying to it with no can $d o,{ }^{21}$ or introducing a colleague as a Python Guru ${ }^{22}$ or a Java Ninja ${ }^{22}$ or complaining my bug reports are falling on deaf ears, ${ }^{24}$ or that the level of effort is too many man-hours. ${ }^{25}$

Many on-line resources may be useful to readers who would be similarly inclined. But, beware. In seeking greater understanding of the history of terms and phrases you thought you knew, you find a lot of misinformation $^{26}$ out there to sift through. In addition, when you are seeking to learn more about any possible negative impacts of a given word or phrase, it's best to seek opinions from experts within the groups most likely impacted. In other words, if a given phrase has potential negative impacts against Blacks, then language experts within the Black community will likely have the the most authoritative guidance. If a given phrase has potential negative impacts against the deaf, then language experts within the deaf community will likely have the most informed guidance.

Finally, in seeking to fix inclusivity bugs, it's important to take care that we don't introduce another kind of problem... excluding the use of perfectly acceptable language for no other reason than out of fear of looking bad or being labeled non-inclusive. A good example is the word master ${ }^{27}$ alone, wholly apart from slave . Hopefully, we all can agree that master/slave ${ }^{28}$ language is not acceptable.

But, there is much less agreement, notably even within the Black community, about the word master alone and with no relation to slave. Many uses of master have no historical roots in oppressive or genocidal systems. These include such terms as mastermind, postmaster, master key, master recording, achieving mastery of a skill and even common tech terms like webmaster and scrum master. This issue garnered much attention when GitHub announced ${ }^{29}$ it would change its default branch name (which most users simply adopt without question) from master to main. In that move it is worth pointing out that the default language of a widely used resource such as git is a significantly greater proliferation potential and represents a qualitatively different situation than the choices individual projects make. Furthermore, in GitHub's move no project was prevented from using master as a git branch name if they chose.

Given the current social justice climate in which we all operate, some readers will feel that inclusive language efforts are nowhere near enough to meet the moment and are really just a distraction ${ }^{28}$ from bigger issues. It's hard to argue with that. Nonetheless, others will feel like such efforts go way too far. While we can acknowledge both perspectives exist, it is worth considering that when we're accustomed to parroting the status quo, pausing to be more inclusive may feel like oppression. ${ }^{30}$ 
Mark C. Miller is a computer scientist supporting the Weapon Simulation and Computing (WSC) program at Lawrence Livermore National Laboratory for more than 30 years. He has recently developed a passion for addressing issues at the nexus of computing and inclusion. Among other things, Mark contributes to VisIt, Silo, HDF5 and IDEAS-ECP. Contact him at miller86@llnl.gov.

\section{Footnotes}

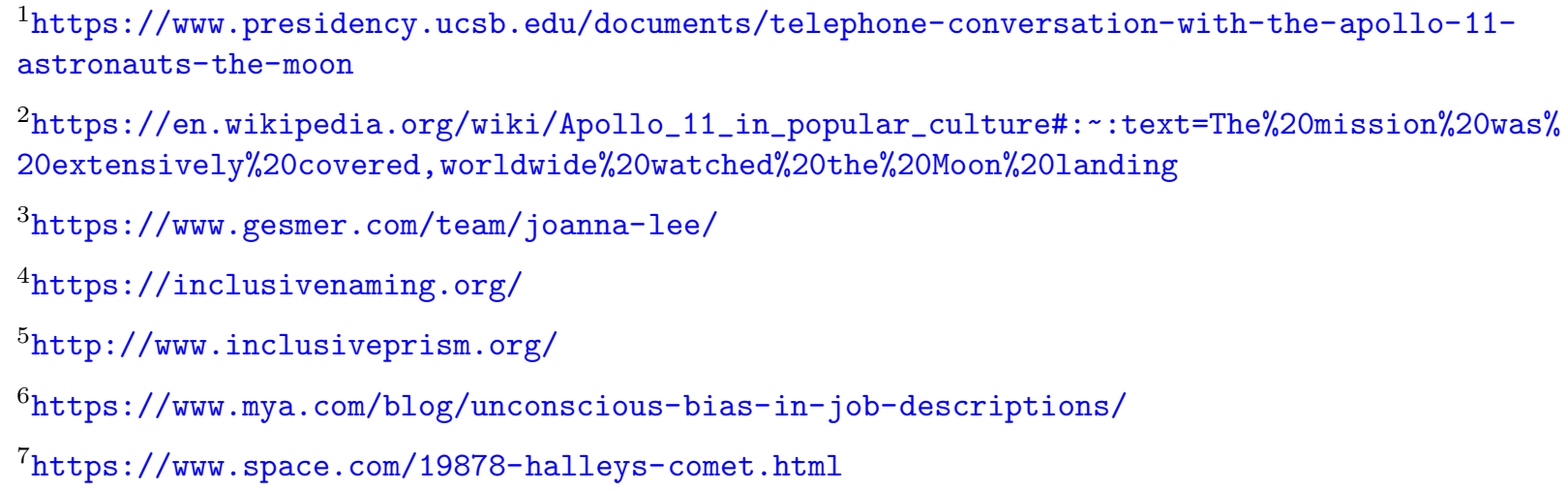

${ }^{8}$ The comet is named for Halley not because he saw or recorded it first but because he is believed to be the first person to have estimated its orbit and based on that connected recorded observations from 1531, 1607 and 1682 as being the same celestial object from which he correctly predicted its return in 1758 . That said, apart from the very significant achievement of the comet's orbital estimation, it isn't clear if astronomers much earlier than Halley had recognized this repeat visitor as the same celestial object.

${ }^{9}$ https://en.wikipedia.org/wiki/Historical_comet_observations_in_China\#Halley's_Comet

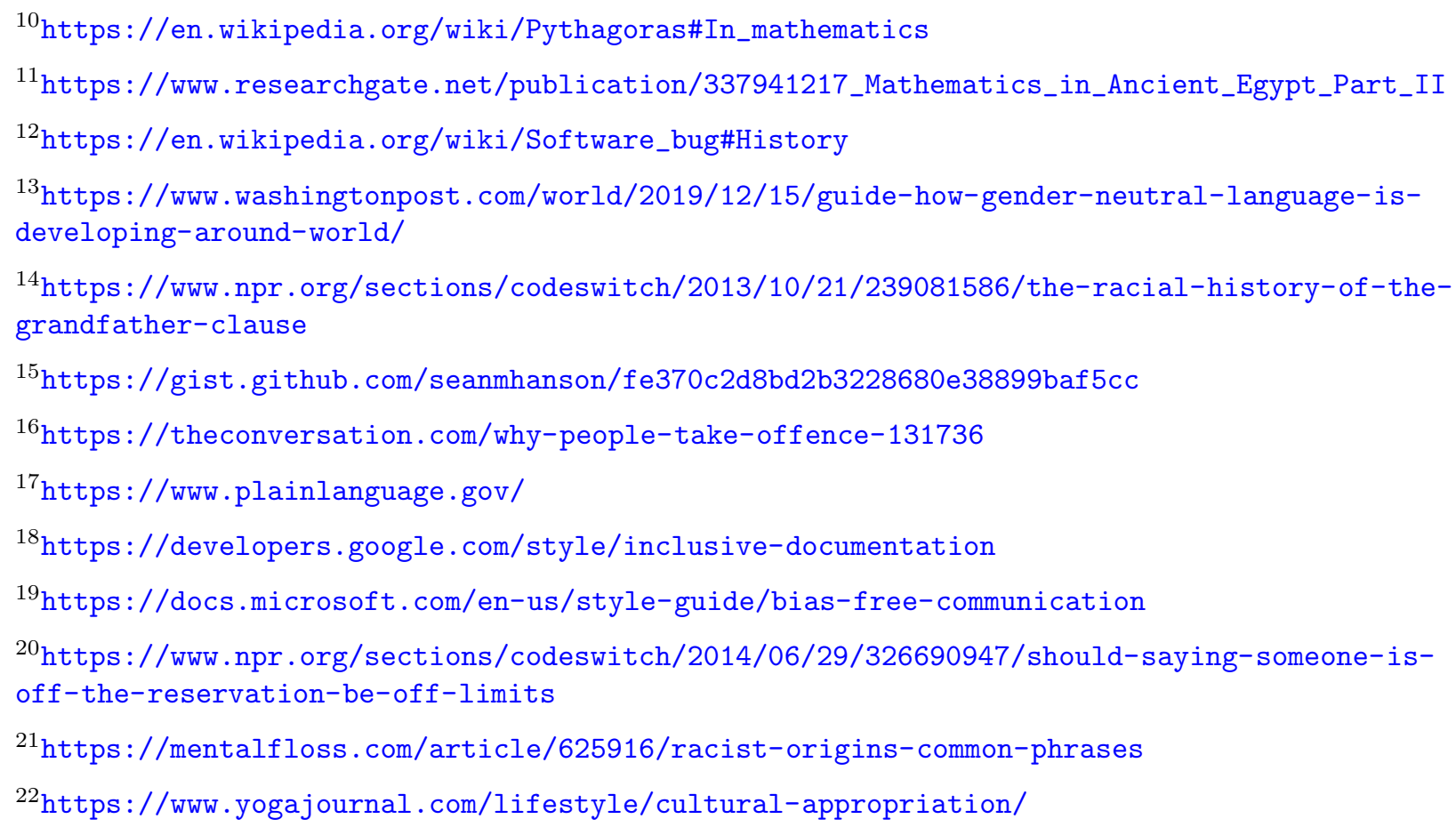


${ }^{24}$ https : //www .hearinglikeme.com/is-the-phrase-falling-on-deaf-ears-offensive/

${ }^{25}$ https : //bossbetty . com/big-story/manpower-man-hours-and-other-phrases-to-ix-nay-fromyour-iased-bay-vocabulary/

${ }^{26}$ https : //www.nytimes.com/1998/01/25/magazine/on-language-misrule-of-thumb.html

${ }^{27}$ https : //www . etymonline.com/word/master

${ }^{28}$ https : //www.wired.com/story/tech-confronts-use-labels-master-slave/

${ }^{29}$ https://www.vice.com/en/article/k7qbyv/github-to-remove-masterslave-terminology-fromits-platform

${ }^{30}$ https ://www.huffpost.com/entry/when-youre-accustomed-to-privilege_b_9460662 